\title{
DPSK Receiver with Implicit Diversity Gain for the Linearly Frequency-Selective Rayleigh Fading Channel
}

\author{
Wing Seng Leon and Desmond P. Taylor \\ Department of Electrical \& Electronic Engineering \\ University of Canterbury \\ Private Bag 4800 \\ Christchurch, New Zealand.
}

\begin{abstract}
This paper presents a simple DPSK receiver for the frequency-selective fading channel. The channel is modelled as an $f$ power series truncated to the first two terms [1], [2]. Two time invariant receiver filters are used to remove the channel induced ISI and to separate the two implicit diversity branches. The received samples from each branch are then differentially decoded and combined. Analytical and simulation results for binary DPSK show that this receiver out performs the conventional receiver using matched filtering and a product demodulator.
\end{abstract}

\section{INTRODUCTION}

The use of differential encoding and detection of PSK signals offers an easy way to circumvent the carrier phase recovery problem caused by fading channels [3]. The receiver is simply a differential decoder and a product detector.

Two problems are generally associated with the reception of DPSK over the frequency-selective fading channel: intersymbol interference (ISI) and symbol to symbol decorrelation of the channel. Due to the delay spread of the channel, the received signal experiences ISI which limits the performance of the conventional DPSK receiver. Even for channels with small delay spread, conventional differential detection yields performance much poorer than that in an equivalent flat-fading channel [4]. In fading channels, the channel parameters vary from symbol to symbol. This results in a performance penalty as the channel estimates provided by the previous received samples are inaccurate [5]. Both these phenomena contribute to an error rate floor. A common technique to decrease the error rate floor is by using explicit diversity [5], [6]. At each sampling instant, the receiver explicitly obtains multiple uncorrelated samples and processes them to obtain diversity gain. This is usually achieved by having several antennas which are spaced far enough apart.

Although a frequency-selective or dispersive fading channel causes ISI in the received signals, it also introduces implicit diversity. Multiple faded and delayed versions of the transmitted signal are received. Like a receiver employing explicit diversity, a receiver able to exploit the implicit channel diversity will improve the error rate performance of the system [7].

In this paper, we propose a simple receiver for DPSK transmitted over a frequency-selective fading channel. The delay spread is assumed to be small enough $(\leq \sim 0.05 T)$ that a two term $f$-power series expansion is sufficient in approximating the channel [1], [2], [8]. This is known as a linearly frequencyselective fading channel. The proposed receiver eliminates the channel induced ISI and also exploits the implicit diversity of the channel. Therefore, it shows better performance than conventional DPSK receivers using matched filters and differential decoders. Like the receivers for strictly non-selective fading channels in [9], [10], the proposed receiver consists of a pair of time invariant receiver filters. These eliminate the ISI from the received signal and separate the two implicit diversity branches present. The sampled outputs from each filter are then differentially decoded and combined to obtain diversity gain prior to detection.

The paper is organized as follows. Section II defines the system model. The design of the receiver is developed in section III. Section IV presents the performance of the proposed receiver for the case of binary DPSK. Conclusions are contained in section $\mathrm{V}$.

\section{Channel and Signal Model}

In an M-ary DPSK system, the M-PSK data symbols

$$
\alpha_{l}=\exp \left(j a_{l}\right)
$$

with $a_{l} \in\left\{b_{m}=2 \pi m / M ; m=0,1, \ldots, M-1\right\}$ are differentially encoded as

$$
\beta_{l}=\alpha_{l} \beta_{l-1} .
$$

The complex baseband transmitted signal may be written as

$$
s(t)=\sum_{l} \beta_{l} p(t-l T)
$$

where $p(t)$ is the impulse response of the transmitter filter and $T$ is the symbol period.

The channel is a time- and frequency-selective Rayleigh fading channel. The time variant impulse response using a conventional tapped delay line (TDL) model is given by

$$
c(\tau, t)=\sum_{i=1}^{I} \phi_{i}(t) \delta\left(\tau-\tau_{i}\right)
$$

where $\phi_{i}(t)$ is the zero mean complex Gaussian random variable associated with the $i$-th ray of the channel. The delay of the $i$-th path of the channel is represented by $\tau_{i}$ and there is a total of $I$ delayed paths. We assume a wide sense stationary uncorrelated scattering (WSSUS) channel.

Instead of a tapped delay line model as in (4), we model the channel as an $f$-power series expansion [1], [2], [8]. For 
channels with small delay spreads, a two term $f$-power series is sufficient in representing the channel [2], [8]. This is known as a linearly frequency-selective fading channel. We also assume that the fading is slow and piecewise constant over each symbol interval [5]. We may then write the received signal as

$$
r(t)=\sum_{l} \psi_{l}^{0} \beta_{l} p(t-l T)+\sum_{l} \psi_{l}^{1} \beta_{l} q(t-l T)+w(t)
$$

with

$$
q(t)=T \frac{d\{p(t)\}}{d t} .
$$

The coefficients $\psi_{l}^{0}$ and $\psi_{l}^{1}$ are zero mean complex Gaussian fading processes for the $l$-th symbol interval associated with the first and second term of the series model respectively. These fading coefficients are related to the tap weights of the TDL model in (4) by [8], [11]

$$
\psi_{l}^{\kappa}=\sum_{i=1}^{I}\left(\frac{-\tau_{i}}{T}\right)^{\kappa} \frac{\phi_{i}(l T)}{\kappa !} .
$$

Each term on the r.h.s. of (5) may be viewed as the output of an implicit diversity branch of the channel. The complex white Gaussian noise present at the front end of the receiver is denoted by $w(t)$ and has power spectral density $N_{0}$. As in [8], we assume that the timing recovery circuit locks onto the mean delay and effectively sets it to zero. The correlation between the fading coefficients becomes

$$
E\left[\psi_{l}^{0} \psi_{k}^{1 *}\right]=0
$$

where $*$ denotes complex conjugation.

\section{THE RECEIVER DESIGN}

\section{A. Equalization and Diversity Combining}

A block diagram of the proposed receiver is shown in fig. 1 . Like the flat-fading receivers of [9], [10], we propose a receiver consisting of two receiver filters with impulse responses $h_{x}(t)$ and $h_{y}(t)$. They serve to minimize the ISI and to separate the two diversity branches. Their outputs at time $t=k T$ are

$x_{k}=\sum_{l} \psi_{l}^{0} \beta_{l} p_{x}([k-l] T)+\sum_{l} \psi_{l}^{1} \beta_{l} q_{x}([k-l] T)+w_{x}(k)$

and

$$
y_{k}=\sum_{l} \psi_{l}^{0} \beta_{l} p_{y}([k-l] T)+\sum_{l} \psi_{l}^{1} \beta_{l} q_{y}([k-l] T)+w_{y}(k)
$$

where

$$
\begin{aligned}
p_{x}(t) & =p(t) \otimes h_{x}(t) \\
q_{x}(t) & =q(t) \otimes h_{x}(t) \\
p_{y}(t) & =p(t) \otimes h_{y}(t) \\
q_{y}(t) & =q(t) \otimes h_{y}(t) \\
w_{x}(k) & =\left[w(t) \otimes h_{x}(t)\right]_{t=k T} \\
w_{y}(k) & =\left[w(t) \otimes h_{y}(t)\right]_{t=k T}
\end{aligned}
$$

and $\otimes$ represents the convolution operator. Ideally, all ISI is removed and the two signal branches are entirely separated. The desired sampled outputs from these filters are therefore

$$
\begin{aligned}
& x_{k}=\psi_{k}^{0} \beta_{k}+w_{x}(k) \\
& y_{k}=\psi_{k}^{1} \beta_{k}+w_{y}(k) .
\end{aligned}
$$

As shown in appendix A, to achieve (17) and (18) the overall responses $P_{x}(f)$ and $P_{y}(f)$ are

$P_{x}(f)=P(f) H_{x}(f)= \begin{cases}T(1+f T) & \text { for } \quad-\frac{1}{T} \leq f \leq 0 \\ T(1-f T) & \text { for } \quad 0 \leq f \leq \frac{1}{T}\end{cases}$

and

$$
P_{y}(f)=P(f) H_{y}(f)=\left\{\begin{array}{ccc}
\frac{j T}{2 \pi} & \text { for } & -\frac{1}{T} \leq f \leq 0 \\
\frac{-j T}{2 \pi} & \text { for } & 0 \leq f \leq \frac{1}{T}
\end{array}\right.
$$

where $P(f), H_{x}(f), H_{y}(f)$ are the filter transfer functions of $p(t), h_{x}(t)$ and $h_{y}(t)$. Note that the use of these filters require $100 \%$ excess bandwidth. The samples in (16) and (17) are then differentially demodulated resulting in

$$
\begin{aligned}
\tilde{\alpha}_{k}^{x} & =x_{k} x_{k-1}^{*} \\
\tilde{\alpha}_{k}^{y} & =y_{k} y_{k-1}^{*}
\end{aligned}
$$

where $\tilde{\alpha}_{k}^{x}$ and $\tilde{\alpha}_{k}^{y}$ are noisy and scaled estimates of the $k$-th data symbol. The samples from each diversity branch are then linearly combined. This is a form of diversity combining. Symbol decisions are made by the decision device using a product detector which computes the decision metric [5]

$$
\Lambda_{k}\left(b_{m}\right)=\operatorname{Re}\left[\left(\tilde{\alpha}_{k}^{x}+\tilde{\alpha}_{k}^{y}\right) \exp \left(-j b_{m}\right)\right]
$$

for all $M$ possible values of $b_{m}$. The detector makes the decision $\alpha_{k}=\exp \left(j b_{l}\right)$ if $b_{l}$ maximizes the metric (23).

Clearly, the receiver will still operate using only one of the two receiver filters. However, the implicit diversity of the channel will not be exploited.

\section{B. Filters Selection and Noise Figures}

There are numerous choices of $P(f), H_{x}(f)$ and $H_{y}(f)$ which satisfy (19) and (20). However, a simple family of solutions arises if we simply partition the frequency response in (19) such that

$$
H_{x}(f)= \begin{cases}{[T(1+f T)]^{n}} & \text { for } \quad-\frac{1}{T} \leq f \leq 0 \\ {[T(1-f T)]^{n}} & \text { for } \quad 0 \leq f \leq \frac{1}{T}\end{cases}
$$

and

$$
P(f)= \begin{cases}{[T(1+f T)]^{1-n}} & \text { for } \quad-\frac{1}{T} \leq f \leq 0 \\ {[T(1-f T)]^{1-n}} & \text { for } \quad 0 \leq f \leq \frac{1}{T}\end{cases}
$$

where the partitioning index $n$ has values in the range $0 \leq n \leq$ 1. Using (20) and the fact that

$$
H_{x}^{\prime}(f)=\left\{\begin{array}{clc}
n T^{2} P^{-1}(f) & \text { for } & -\frac{1}{T} \leq f \leq 0 \\
-n T^{2} P^{-1}(f) & \text { for } & 0 \leq f \leq \frac{1}{T}
\end{array}\right.
$$


where $H_{x}^{\prime}(f)$ is the first derivative of $H_{x}(f)$ with respect to the frequency variable $f$, we write the transfer function of the second filter as

$$
H_{y}(f)=\frac{j H_{x}^{\prime}(f)}{2 \pi n T}
$$

Hence, the impulse responses of the filters $h_{x}(t)$ and $h_{y}(t)$ are related by

$$
h_{y}(t)=\frac{t h_{x}(t)}{n T} .
$$

As shown in (15) and (16), the AWGN is shaped by the two receiver filters. Therefore, the choice of partitioning index also effects the noise statistics. In general the noise correlation function is expressed as [12]

$$
\begin{aligned}
& E\left[w_{u}(t) w_{v}^{*}(t+\xi)\right]=R_{u v}(\xi) \\
& \quad=N_{0} \int_{-\infty}^{\infty} H_{u}(f) H_{v}^{*}(f) \exp (j 2 \pi f \xi) d f .
\end{aligned}
$$

For the autocorrelation of $w_{x}(t), u=v=x$ and for the autocorrelation of $w_{y}(t), u=v=y$. In the case of the crosscorrelation between the two noises, we let $u=x$ and $v=y$.

Using (24) and (27), we evaluated the integral in (29) for $R_{y y}(0)$, the variance of the noise $w_{y}(t)$. It turns out that $R_{y y}(0)$ does not converge for any values of $n \leq 1 / 2$. Therefore, the only acceptable values of the partitioning index are in the range $1 / 2<n \leq 1$. Choosing otherwise will cause a large amplification of noise from the output of filter $h_{y}(t)$. However, by using the allowable values of $n$, the filtered noise samples become correlated. Using (24), (26), (27) and (29), the noise variances are expressed as

$$
R_{x x}(0)=\frac{2 N_{0} T^{2 n-1}}{2 n+1}
$$

and

$$
R_{y y}(0)=\frac{N_{0} T^{2 n-1}}{2 \pi^{2}(2 n-1)}
$$

for $1 / 2<n \leq 1$. It is obvious from (30) and (31) that as the partition index $n$ increases, the noise variances at the outputs of the filters decreases.

In the special case of $n=1$, the transmitter filter becomes an ideal low pass filter between $-1 / T \leq 0 \leq 1 / T$ and therefore all pulse shaping is done at the receiver. The impulse responses of the receiver filters are then $h_{x}(t)=\operatorname{sinc}^{2}(t / T)$ and $h_{y}(t)=$ $t T^{-1} \operatorname{sinc}^{2}(t / T)$.

\section{Receiver Performance}

The performance of the receiver was evaluated by analysis and computer simulation for various channel parameters. Binary DPSK was used as the modulation scheme and the average bit energy is denoted by $E_{b}$. A channel with two delayed paths was used. The channel was assumed to have an equal power split where the average power associated with the first and second rays are equal. We assume that the fading process

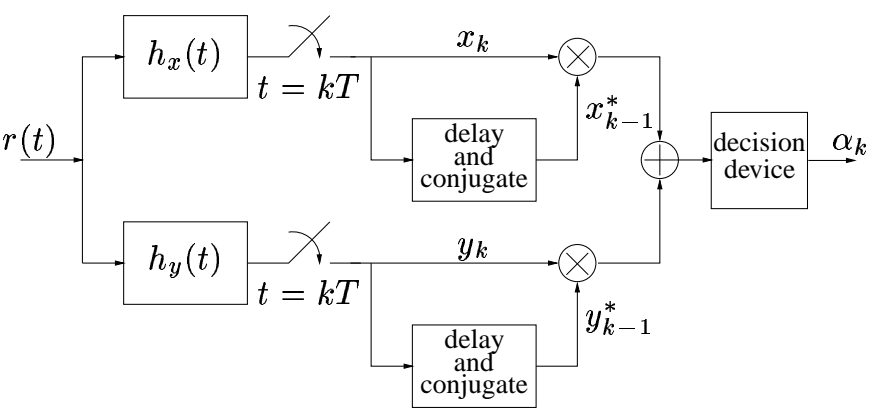

Fig. 1. Block diagram of the proposed receiver.

associated with each path, $\phi_{i}(t)$ has a normalized autocorrelation function $R_{\phi \phi}(\xi)=J_{0}\left(2 \pi f_{d} \xi\right)$ [13]. $J(\bullet)$ is the zero order Bessel function of the first kind and $f_{d}$ is the maximum Doppler bandwidth. The transmitter and receiver filters used have transfer functions as shown in (24), (25) and (27) with partitioning index of $n=0.75$. The results are presented as bit error probability, $P_{b}$ versus $E_{b} / N_{0}$ plots and are shown in fig. 2 to 5 .

\section{A. Analytical Results}

To evaluate the performance of the receiver, we compute its bit error probability. Given that the transmitted symbol is $\alpha_{k}=$ $\exp \left(j b_{m}\right)$, the receiver makes an error if it decides on symbol $\hat{\alpha}_{k}=\exp \left(j \hat{b}_{m}\right)$ where $\alpha_{k} \neq \hat{\alpha}_{k}$. Using (21) to (23), we define the random variable $D$ as

$$
\begin{aligned}
& D=\Lambda\left(b_{m}\right)-\Lambda\left(\hat{b}_{m}\right) \\
& =\frac{1}{2}\left[x_{k} x_{k-1}^{*} d_{k}^{*}+y_{k} y_{k-1}^{*} d_{k}^{*}+x_{k}^{*} x_{k-1} d_{k}+y_{k}^{*} y_{k-1} d_{k}\right]
\end{aligned}
$$

where

$$
d_{k}=\alpha_{k}-\hat{\alpha}_{k}=\exp \left(j b_{m}\right)-\exp \left(j \hat{b}_{m}\right) .
$$

An error event occurs when random variable $D<0$. For any given pair, $\alpha_{k}$ and $\hat{\alpha}_{k}$, the probability of $D<0$ or the pairwise probability of error is written as

$$
P\left(\alpha_{k} \rightarrow \hat{\alpha}_{k}\right)=\operatorname{Prob}(D<0) .
$$

The expression in (32) is a quadratic form and the pairwise probability of error in (34) is evaluated using the eigenvalue technique in [14], [15]. Since we are using binary DPSK and we assume equiprobable symbols, the average bit error probability $P_{b}$ is simply the average pairwise probability of error for the two possible values of $\alpha_{k}$.

For comparison, we have included analytical results obtained using a conventional matched filter using square-root raised cosine pulse shape with $100 \%$ roll off obtained in a similar method as in [4].

Fig. 2, shows the results for fade rate $f_{d} T=0.01$ and rms delay spread of $0.05 T$. The error floor is reduced from $1.5 \times 10^{-3}$, for DPSK using a conventional matched filter, to $5 \times 10^{-4}$ using a single equalization filter $h_{x}(t)$. By employing both filters $h_{x}(t), h_{y}(t)$ and linear combining of the demodulated outputs, the error floor is further reduced to about $10^{-5}$. 
Fig. 3 shows the results for fade rate of $f_{d} T=0.005$ and rms delay spread of $0.05 T$. The error floor is reduced from $1.2 \times 10^{-3}$ to $1.2 \times 10^{-4}$ and $6 \times 10^{-7}$ by using a single equalization filter and both filters with diversity combining respectively. For fade rate of $f_{d} T=0.001$ and rms delay spread of $0.05 T$ as shown in fig. 4, the error floor using conventional matched filtering is about $1.1 \times 10^{-3}$. The floor is reduced to $5 \times 10^{-6}$ and about $10^{-9}$ using the single $h_{x}(t)$ filter and both filters with diversity combining respectively.

The above analytical results illustrate the substantial improvement in the error floors by using the proposed receiver. With the use of only a single filter $h_{x}(t)$, some performance improvement was observed. With the use of both filters, the error floors were further lowered due to the use of the implicit diversity present. However, as the fade rate increases, the improvement in the error performance decreases.

\section{B. Simulation Results}

Computer simulations of the proposed receiver with twin filters were used to verify the analytical results of section IV-A. The fading process of each ray was generated as in [16]. The partitioning index used was $n=0.75$.

The simulated $P_{b}$ versus $E_{b} / N_{0}$ plots are shown in fig. 5 for the different fade rates and rms delay $\tau_{r m s}=0.05 T$. For comparison, the analytical curves for the receiver are also plotted as solid lines in fig. 5. For the range of $E_{b} / N_{0}$ values considered, the simulated results agree closely with the analytical results.

\section{CONCLUSION}

A new DPSK receiver for the linearly frequency-selective channel has been presented. The receiver mitigates the ISI induced by the dispersive channel and also exploits the implicit diversity present in the received signal. This is achieved by using transmitter and receiver filters with $100 \%$ excess bandwidth specially designed for these purposes. Analytical and simulation results have shown that the proposed receiver has better error performance than the conventional DPSK receiver using matched filters.

\section{APPENDIX A}

To achieve the desired samples (17) and (18), the following conditions must be met

$$
\begin{aligned}
p_{x}(k T) & =\delta_{k 0} \\
q_{x}(k T) & =0 \\
p_{y}(k T) & =0 \\
q_{y}(k T) & =\delta_{k 0}
\end{aligned}
$$

where the function $\delta_{k 0}=1$ for $k=0$ and is zero otherwise. Thus, the filter responses $p_{x}(t)$ and $q_{y}(t)$ must be ISI free. Also, the filter responses $p_{y}(t)$ and $q_{x}(t)$ must be zero at all sampling epochs. In order to design filters $p(t), h_{x}(t)$ and $h_{y}(t)$ meeting conditions (35) to (38), we follow the method in [9]. We begin by applying the Poisson sum formula [17] to (35) and (36) resulting in

$$
\begin{aligned}
& \sum_{k} P_{x}\left(f-\frac{k}{T}\right)=T \\
& \sum_{k} Q_{x}\left(f-\frac{k}{T}\right)=0
\end{aligned}
$$

where $P_{x}(f), Q_{x}(f)$ are the Fourier transforms of $p_{x}(t)$ and $q_{x}(t)$ respectively. Condition (39) is equivalent to the Nyquist criterion for ISI-free transmission [12]. We also note that the Fourier transforms of the filter responses are given by

$$
\begin{aligned}
P_{x}(f) & =P(f) H_{x}(f) \\
Q_{x}(f) & =j 2 \pi T f P(f) H_{x}(f)
\end{aligned}
$$

where $P(f)$ and $H_{x}(f)$ are the filter transfer functions of $p(t)$ and $h_{x}(t)$ respectively. Assuming that the filters are bandlimited to $-1 / T \leq f \leq 1 / T$ we write (39) and (40) as

$$
\begin{gathered}
P(f) H_{x}(f)+P\left(f-\frac{1}{T}\right) H_{x}\left(f-\frac{1}{T}\right)=T \\
\left(\begin{array}{l}
j 2 \pi T f P(f) H_{x}(f) \\
+j 2 \pi T\left(f-\frac{1}{T}\right) P\left(f-\frac{1}{T}\right) H_{x}\left(f-\frac{1}{T}\right)
\end{array}\right)=0
\end{gathered}
$$

for $0 \leq f \leq 1 / T$. Solving these equations we obtain

$$
P(f) H_{x}(f)=T(1-f T) .
$$

Similarly, for the negative frequencies $-1 / T \leq f \leq 0$, we get

$$
P(f) H_{x}(f)=T(1+f T) .
$$

The solutions shown in (45) and (46) are equivalent to the ones presented in [2] obtained using a more tedious calculus of variation technique. The above approach is applied to (37) and (38) and we get

$$
P(f) H_{y}(f)=\left\{\begin{array}{clc}
\frac{j T}{2 \pi} & \text { for } & -\frac{1}{T} \leq f \leq 0 \\
\frac{-j T}{2 \pi} & \text { for } & 0 \leq f \leq \frac{1}{T}
\end{array}\right.
$$

The overall responses (45) to (47), of the filters $P(f), H_{x}(f)$ and $H_{y}(f)$ must be achieved to meet conditions (35) to (38).

\section{REFERENCES}

[1] P.A. Bello, "Characterization of randomly time-variant linear channels," IEEE Trans. Commun. Sys., vol. 11, pp. 360-393, Dec. 1963.

[2] W.S. Leon, U. Mengali, and D.P. Taylor, "Equalization of linearly frequency selective fading channels," IEEE Trans. Commun., vol. 45, pp. 1501-1503, Dec. 1997.

[3] J.G. Proakis, Digital Communications, McGraw Hill, New York, third edition, 1995.

[4] W. Liu and P. Ho, "Differential detection of psk signals in frequency selective rayleigh fading channel," in Proc. of GLOBECOM, Orlando, Florida, 1992, vol. 2, pp. 1802-1806.

[5] P.Y. Kam, "Bit error probabilities of mdpsk over the nonselective rayleigh fading channel with diversity reception,' IEEE Trans. Commun., vol. 39, pp. 220-224, Feb. 1991. 


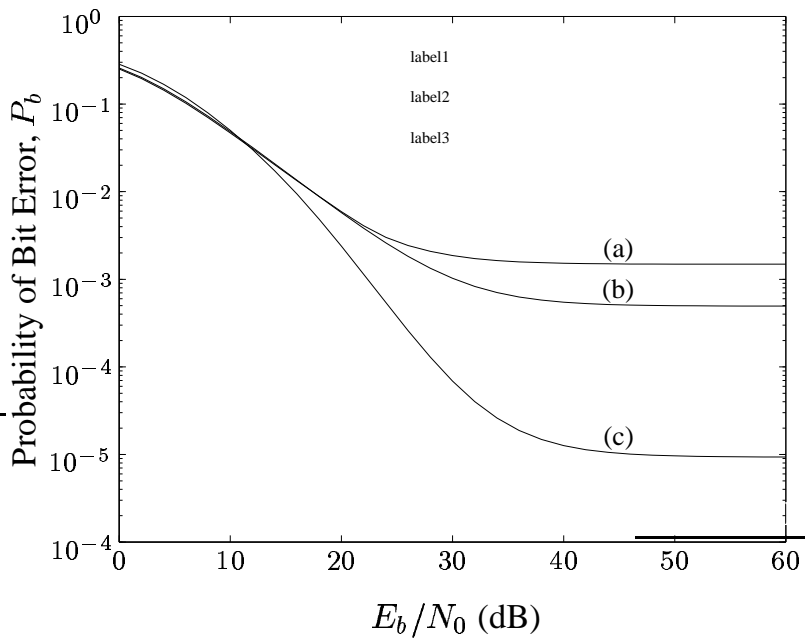

Fig. 2. Analytical probability of error curves for $f_{d} T=0.01$ and $\tau_{r m s}=$ $0.05 T$, binary DPSK with (a) matched filtering using $100 \%$ roll off root raised cosine pulse shape, (b) a single equalization filter $h_{x}(t), n=0.75$ and $(c)$ hoth enualization and diversitv filters. $n=0.75$.

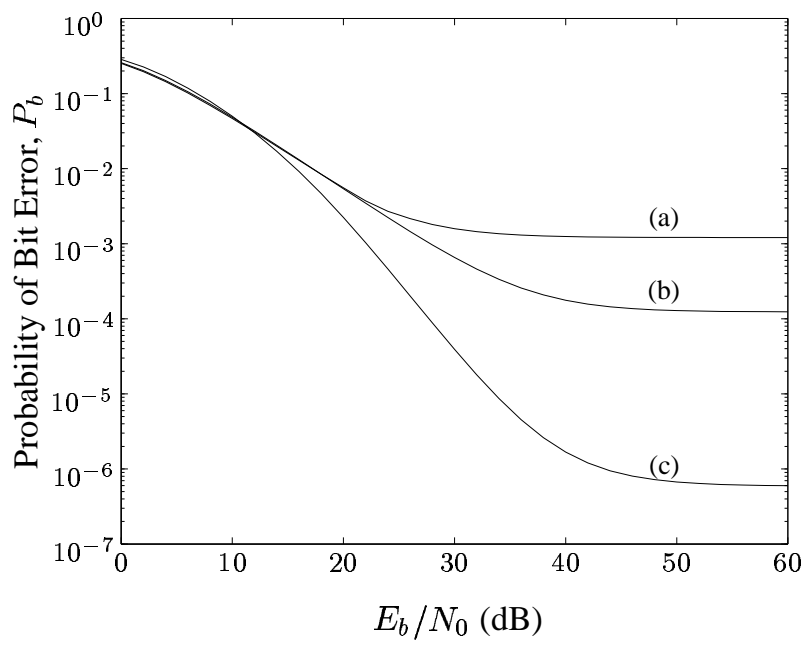

Fig. 3. Analytical probability of error curves for $f_{d} T=0.005$ and $\tau_{r m s}=$ $0.05 T$, binary DPSK with (a) matched filtering using $100 \%$ roll off root raised cosine pulse shape, (b) a single equalization filter $h_{x}(t), n=0.75$ and (c) both equalization and diversity filters, $n=0.75$.

[6] D. Noneaker and M. Pursley, "M-ary differential phase-shift keying with diversity combining for communications over a doubly selective fading channel," in Proc. of Int'l Conf. on Communications, Chicago, Illinois, 1992, vol. 1, pp. 302.5.1-5.

[7] B. Hart and D.P. Taylor, "Extended mlse diversity receiver for the timeand frequency-selective channel," IEEE Trans. Commun., vol. 45, pp. 322-333, Mar. 1997.

[8] G. Deng, J. Cavers, and P. Ho, “A reduced dimensionality propagation model for frequency selective rayleigh fading channels," in Proc. of Int'l Conf. on Communications, Seattle, 1995, pp. 1158-1162.

[9] G.M. Vitetta, D.P. Taylor, and U. Mengali, "Double-filtering receiver for psk signals transmitted over rayleigh frequency-flat fading channels," IEEE Trans. Commun., vol. 44, pp. 686-695, June 1996.

[10] G.M. Vitetta, U. Mengali, and D.P. Taylor, "Differential detection of psk signals transmitted over linearly time-selective rayleigh fading channels," in Proc. of Int'l Conf. on Communications, Atlanta, Georgia, 1998, vol. 1, pp. 433-437.

[11] W.S. Leon and D.P. Taylor, "Mlse receiver for the dispersive rayleigh

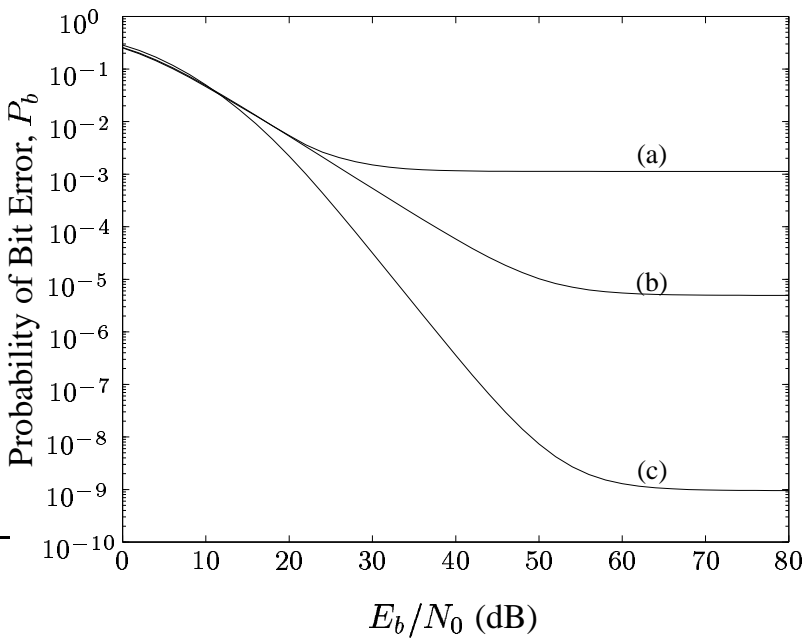

Fig. 4. Analytical probability of error curves for $f_{d} T=0.001$ and $\tau_{r m s}=$ $0.05 T$, binary DPSK with (a) matched filtering using $100 \%$ roll off root raised cosine pulse shape, (b) a single equalization filter $h_{x}(t), n=0.75$ and $(c)$ hoth enualization and diversitv filters. $n=0.75$

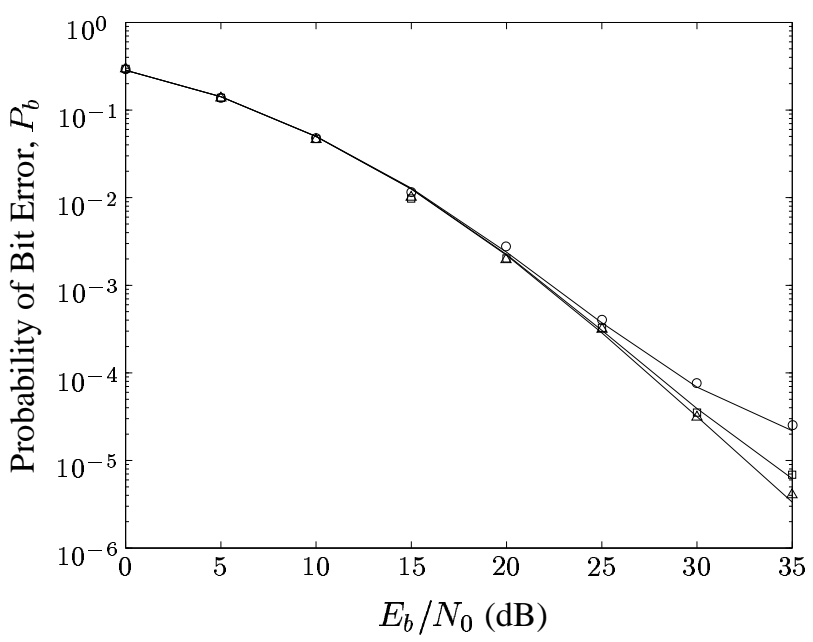

Fig. 5. Simulated and analytical probability of error curves for various $f_{d} T$ and $\tau_{r m s}=0.05 T$ using both filters with $n=0.75$. Analytical results are plotted in solid lines. The marker o represents the simulated results for $f_{d} T=0.01, \square$ for $f_{d} T=0.005$ and $\triangle$ for $f_{d} T=0.001$.

fading channel," in Proc. of Int'l Conf. on Communications, Montreal, Canada, June 1997, vol. 3, pp. 1513-1517.

[12] S. Haykin, Communication Systems, John Wiley and Sons, New York, third edition, 1994.

[13] W.C. Jakes Jr., Microwave Mobile Communications, John Wiley \& Sons, New York, 1974.

[14] M. Schwartz, W.R. Bennett, and Seymour Stein, Communication Systems and Techniques, McGraw-Hill, New York, 1966.

[15] M.J. Barrett, "Error probability for optimal and suboptimal quadratic receivers in rapid rayleigh fading channels," IEEE J. Select. Areas Commun., vol. 5, pp. 302-304, Feb. 1987.

[16] D. Verdin and T.C. Tozer, "Generating a fading process for the simulation of land-mobile radio communications," Electronics Letters, vol. 29, no. 23, pp. 2011-2012, Nov. 1993.

[17] A. Papoulis, Probability, Random Variables, and Stochastic Processes, McGraw-Hill, New York, third edition, 1991. 